See discussions, stats, and author profiles for this publication at: https://www.researchgate.net/publication/335152822

\title{
Orbits of maximal invariant subgroups and solvability of finite groups
}

Article in Journal of Algebra · August 2019

DOI: 10.1016/j.jalgebra.2019.08.006

\section{CITATIONS}

0

2 authors:

Changguo Shao

University of Jinan (Jinan, China)

41 PUBLICATIONS 176 CITATIONS

SEE PROFILE
READS

42

Antonio Beltrán

Universitat Jaume I

65 PUBLICATIONS 287 CITATIONS

SEE PROFILE

Some of the authors of this publication are also working on these related projects:

Relations between the product of conjugacy classes and the solvability and non-simplicity of finite groups View project

Invariant structure of finite groups View project 


\title{
ORBITS OF MAXIMAL INVARIANT SUBGROUPS AND SOLVABILITY OF FINITE GROUPS
}

\author{
CHANGGUO SHAO \\ SCHOOL OF MATHEMATICAL SCIENCE \\ UNIVERSITY OF JINAN \\ 250022 SHANDONG \\ CHINA \\ ANTONIO BELTRÁN \\ DEPARTAMENTO DE MATEMÁTICAS \\ UNIVERSITAT JAUME I \\ 12071 CASTELLÓN \\ SPAIN
}

\begin{abstract}
Let $A$ and $G$ be finite groups having coprime orders and suppose that $A$ acts on $G$ via automorphisms. We give some solvability criteria for $G$ according to the number of orbits that appear by the action of the fixed point subgroup $\mathbf{C}_{G}(A)$ on the set of maximal $A$-invariant subgroups of $G$, and likewise, on the set of non-nilpotent maximal $A$-invariant subgroups. We also obtain some characterizations and further structure properties of these groups. In the course of our study we prove an independent result concerning maximal factorizations of classical simple groups.
\end{abstract}

\section{INTRODUCTION}

A topic of interest in Finite Group Theory is to investigate the structure and properties of a group $G$ under certain conditions on its maximal subgroups. An elementary result claims that a finite group all whose maximal subgroups are conjugate must be necessarily a cyclic group of prime power order. In 1980, S. Adnan proved by elementary methods that when $G$ has exactly two conjugacy classes of maximal subgroups then $G$ is solvable. In fact, the order of $G$ is divisible by exactly two primes and it has a very specific structure ([1], [2]). However, groups having three or more conjugacy classes of maximal subgroups need not be solvable. For instance, there exist simple groups such as $\operatorname{PSL}(2,7)$ that have exactly three conjugacy classes of maximal subgroups, two of which are interchanged by an outer automorphism of order 2. Afterwards, once the Classification of the Finite Simple

2010 Mathematics Subject Classification. 20E28, 20D06, 20D45, $20 \mathrm{D} 15$.

Key words and phrases. Maximal invariant subgroups, coprime action, maximal subgroups of simple groups of Lie type.

Changguo Shao: shaoguozi@163.com; Antonio Beltrán: abeltran@uji.es

The authors would like to thank C.H. Li for many useful suggestions. The first author is supported by the Nature Science Fund of Shandong Province (No. ZR2019MA044) and the Opening Project of Sichuan Province University Key Laboratory of Bridge Non-destruction Detecting and Engineering Computing(No.2018QZJ04). The second author is partially supported by Ministerio de Ciencia, Innovación y Universidades, Proyecto PGC2018-096872-B-100. 
Groups (CFSG) was completed, V.A. Belonogov gave a precise description of such groups (see [3]).

In this paper we pose a more general hypothesis. We assume that a finite group $A$ is acting coprimely on a group $G$ by automorphisms, that is, $(|G|,|A|)=1$, and we focus on the set of maximal $A$-invariant subgroups of $G$. Now, conjugates of maximal $A$-invariant subgroups of $G$ need not be $A$-invariant, that is, in general $G$ does not act on the set of maximal $A$-invariant subgroups. But the fixed point subgroup, $\mathbf{C}_{G}(A)$, which usually plays a crucial role in the coprime action setting, does act on this set. Accordingly, there is sense in studying the case in which there are few $\mathbf{C}_{G}(A)$-orbits of maximal $A$-invariant subgroups in $G$. We demonstrate the solvability of $G$ and determine its structure when there only exist at most two orbits.

Our first result, which is precise and elementary, deals with the case of just one orbit. When all maximal $A$-invariant subgroups of $G$ are conjugate by some element in $\mathbf{C}_{G}(A)$, then $G$ is not necessarily a cyclic (or abelian) group of prime power order, as it happens when $A=1$. We prove the following characterization in Section 2 .

Theorem A. Suppose that a finite group $A$ acts coprimely on a finite group $G$. Then all maximal $A$-invariant subgroups of $G$ are $\mathbf{C}_{G}(A)$-conjugate if and only if $G$ is a p-group and $A$ acts irreducibly on $G / \Phi(G)$. In this case, $G$ has exactly one maximal $A$-invariant subgroup, which is $\Phi(G)$.

Unlike Adnan's work, the case of two orbits of maximal invariant subgroups is much more complex. Once we manage to reduce the problem to simple groups, we need to appeal to the CFSG for studying those maximal factorizations in a simple group of Lie type in which both maximal subgroups have coprime indexes. This is previously done in Section 3 with a case-by-case analysis on the maximal factorizations of the finite simple groups of Lie type, which are given in [11] (classical groups) and [13] (exceptional groups). We think that Theorem 3.4 is of particular interest.

Before stating our main result, whose proof is given in Section 4, we will establish some notation. For every prime $p$, we will denote by $\operatorname{Syl}_{p}^{A}(G)$ the set of $A$-invariant Sylow $p$-subgroups of $G$. We recall that this set is not empty by elementary coprime action properties, and that any two subgroups lying in $\operatorname{Syl}_{p}^{A}(G)$ are $\mathbf{C}_{G}(A)$-conjugate.

Theorem B. Suppose that a finite group A acts coprimely on a finite group $G$. If all maximal $A$-invariant subgroups of $G$ lie in two $\mathbf{C}_{G}(A)$-orbits, then $G$ is solvable and one of the following conditions holds:

(1) $G$ is a prime power order group.

(2) $G=P Q$, where $P \in \operatorname{Syl}_{p}^{A}(G)$ and $Q \in \operatorname{Syl}_{q}^{A}(G)$, and $P \unlhd G$. In addition, $P / \Phi(P)$ is $Q A$-irreducible, that is, $P / \Phi(P)$ has no proper non-trivial $Q A$-invariant subgroup.

Taking one more step, it was recently proved that if a group $G$ has a single conjugacy class of non-nilpotent maximal subgroups, then $G$ is solvable. This is an immediate consequence of Theorem 1.2 of [15]. Likewise, it has been shown that if $G$ has at most two conjugacy classes of non-nilpotent maximal subgroups, then $G$ is solvable as well (Theorem 
1.1 of [14]). Both results require the CFSG. We wonder whether these properties can be transferred into the coprime action scenario and give a positive answer in Section 5 .

Theorem C. Suppose that a group $A$ acts coprimely on a group $G$. If $G$ has at most two $\mathbf{C}_{G}(A)$-orbits of non-nilpotent maximal A-invariant subgroups, then $G$ is solvable.

\section{Proof of Theorem A}

We present some results that will be needed for proving our main results, but first we make an observation. The hypotheses in our main theorems are inherited by $A$-invariant quotients. Indeed, if $N \unlhd G$ is $A$-invariant, then the obvious inclusion $\mathbf{C}_{G}(A) N / N \leq$ $\mathbf{C}_{G / N}(A)$ is sufficient for this purpose, and of course this inclusion is not related to the coprimality of the action. Regarding coprime action we state two standard properties. They will be applied in the framework of solvable groups although the statements and proofs are easily extensible to $p$-solvable groups. We refer to [12, Chapter 8] for a detailed presentation and other basic properties of coprime action.

Lemma 2.1. [5, Lemma 2.3] Let $A$ be a group acting coprimely on a $p$-solvable group $G$. If $H$ is a maximal $A$-invariant subgroup of $G$, then $|G: H|$ is a $p$-number or a $p^{\prime}$-number.

Lemma 2.2. Let $A$ be a group acting coprimely on a $p$-solvable group $G$. If $H$ is a maximal $A$-invariant normal subgroup of $G$, then $|G: H|$ is a $p$-number or a $p^{\prime}$-number.

Proof. Since the semidirect product $G A$ is $p$-solvable and $G / H$ is a chief factor of it, $G / H$ is either a $p$-group or a $p^{\prime}$-group.

The fact of using inductive arguments to obtain the solvability in Theorem B forces us to prove first the solvability of a group when it possesses only one orbit of maximal invariant subgroups. So we start by proving Theorem A, whose proof is elementary.

Proof of Theorem $A$. Assume that all maximal $A$-invariant subgroups of $G$ are $\mathbf{C}_{G}(A)$ conjugate. We prove first that $G$ is a $p$-group. Suppose on the contrary that $|G|$ is divisible by more than one prime. Then, for some prime $p$, we take $P \in \operatorname{Syl}_{p}^{A}(G)$ and there exists a maximal $A$-invariant subgroup $M$ of $G$ such that $P \leq M$. Now, $|G: M|$ must be divisible by at least one prime $q \neq p$, and then we choose $N$ to be a maximal $A$-invariant subgroup of $G$ containing an $A$-invariant Sylow $q$-subgroup of $G$. We certainly have that $M$ and $N$ cannot be conjugate, since they have different order, contradicting the hypotheses.

For the second part, let us consider $G / \Phi(G)$, which is also acted on by $A$ and is an elementary abelian $p$-group for some prime $p$. Maschke Theorem $([12,8.4 .6])$ asserts that the action of $A$ on $G / \Phi(G)$ is semisimple. On the other hand, the hypotheses imply that all maximal $A$-invariant subgroups of $G / \Phi(G)$ are $\mathbf{C}_{G}(A)$-conjugate, so by joining both facts, we deduce that $G / \Phi(G)$ has no proper non-trivial $A$-invariant subgroup, that is, $A$ acts irreducibly on $G / \Phi(G)$, as required.

Conversely, assume that $G$ is a $p$-group and that $A$ acts irreducibly on $G / \Phi(G)$. It is straightforward that $\Phi(G)$ is maximal $A$-invariant in $G$. Let $M$ be another maximal $A$-invariant subgroup of $G$. The fact that $\Phi(G)$ is $A$-invariant and the maximality of $M$ imply that $G=M \Phi(G)$. However, $M$ is contained in some maximal subgroup of $G$, say $M_{1}$, and then $G=M_{1} \Phi(G)=M_{1}$, a contradiction. So there exists a single maximal $A$-invariant subgroup in $G$, which is $\Phi(G)$. 
Remark 2.3. Under the hypotheses of Theorem A, if $C:=\mathbf{C}_{G}(A)<G$, then $C \leq \Phi(G)$. If on the contrary $C=G$, then $G$ must be cyclic of prime order.

\section{Maximal factorizations With Subgroups of COPRIME INDEXES IN Simple GROUPS OF LIE TYPE}

For dealing with the two-orbits case, we need to study factorizations in finite simple groups of Lie type, when both factors have coprime indexes. This fact quickly leads to be able to exclude exceptional groups of Lie type. Furthermore, we will see that the fact of introducing coprime action on a simple group lead us to be able to exclude the case in which the defining field of the group has order $p^{f}$ with $p$ prime and $f$ a power of 2 . Let $\hat{L}$ be a classical linear group on vector space $V$ and centre $Z$ (so that $L=\hat{L} / Z$ is a classical simple group), and let $\hat{G}$ be a group such that $\hat{L} \unlhd \hat{G} \leq G L(V)$. For a subgroup $X$ of $\hat{G}$, we denote by ${ }^{\wedge} X$ the $\operatorname{subgroup}(X Z \cap \hat{L}) / Z$ of $L$. Let $d=|Z|$. We also denote by $\frac{1}{d} M$ the factor group of $M Z / Z$ in $L$. As we said in the Introduction, our discussion is based and follows the notation and terminology of [11] and [13].

Lemma 3.1. ([13, Theorem A]) Let $G$ be finite classical simple group of Lie type defined over the field $F$ of order $p^{f}$ with $p$ a prime. Assume that $f$ is not a power of 2. If $G=M N$ is a nontrivial factorization of $G$, then interchanging $M$ and $N$ if necessary, the triple $(G, M, N)$ lies in Tables 2-7 in the Appendix.

Lemma 3.2. ([10, Lemma 3]) Let $a, m$ and $n$ be natural numbers. Then

(a) $\left(a^{m}-1, a^{n}-1\right)=a^{(m, n)}-1$,

(b) $\left(\left(a^{n}-1\right) /\left(a^{(m, n)}-1\right), a^{m}-1\right)=\left(n /(m, n), a^{(m, n)}-1\right)$.

Lemma 3.3. ([18, Lemma 6]) Let $a, s, t$ be positive integers. Then

(a) $\left(a^{s}+1, a^{t}+1\right)=\left\{\begin{array}{l}a^{(s, t)}+1, \text { if both } s /(s, t) \text { and } t /(s, t) \text { are odd; } \\ (2, a+1) \text {, otherwise. }\end{array}\right.$

(b) $\left(a^{s}-1, a^{t}+1\right)=\left\{\begin{array}{l}a^{(s, t)}+1, \text { if } s /(s, t) \text { is even and } t /(s, t) \text { is odd; } \\ (2, a+1) \text {, otherwise. }\end{array}\right.$

Theorem 3.4. Let $G$ be a non-abelian simple group of Lie type over a field of order $q$. Let $q=p^{f}$ with $p$ a prime and $f$ not power of 2. If $M$ and $N$ are subgroups of $G$ with $(|G: M|,|G: N|)=1$, then the triple $(G, M, N)$ (interchanging $M$ and $N$ if necessary) lies in Table 1 below.

Proof. Since $(|G: N|,|G: M|)=1$, we have $G=M N$. Moreover, there must be maximal subgroups $A$ and $B$ satisfying $M \leq A$ and $N \leq B$. Trivially, $G=A B$. In the proof of this theorem, we will replace $M$ and $N$ by such $A$ and $B$ to check the condition $(|G: M|,|G: N|)=1$ many times appealing to [11].

By [13, Theorem B], we may conclude that $G$ is not an exceptional simple group of Lie type by calculation. Therefore, $G$ can be assumed to be a classical simple group of Lie type, that is, $G$ is isomorphic to $P S L_{n}(q), P S p_{2 m}(q), U_{2 m}(q), \Omega_{2 m+1}(q)$ or $P \Omega_{2 m}^{ \pm}(q)$ by $[13$, Theorem A]. We do a case-by-case analysis following Lemma 3.1 which consists of checking that in all the cases that not appear in Table 2, the indexes of $M$ and $N$ are not coprime. 
TABle 1 . Triple $(G, M, N)$

\begin{tabular}{|l|l|l|l|}
\hline$G$ & $M$ & $N$ & Remark \\
\hline$P S L_{n}(q)$ & ${ }^{\wedge} G L_{a}\left(q^{b}\right) \cdot b$ & $P_{1}=q^{n-1}: G L_{n-1}(q) \cong P_{n-1}$ & $\begin{array}{l}b \text { is a prime and } a b=n=b^{s}, \\
b \nmid(q-1), d=(n, q-1)=1\end{array}$ \\
\hline$P S L_{n}(q)$ & $P S p_{n}(q) \cdot a$ & $P_{1}=q^{n-1}: G L_{n-1}(q) \cong P_{n-1}$ & $\begin{array}{l}n=2^{s}, s \geq 2 \text { and } q \text { is even, } \\
d=(n, q-1)=1, a=1 \text { or } 2\end{array}$ \\
\hline$P S p_{2 m}(q)$ & $O_{2 m}^{-}(q)$ & $P_{m}=q^{m(m+1) / 2}: G L_{m}(q)$ & $\begin{array}{l}m>2 \text { is odd, } q \text { is even, } d= \\
(2, q-1)=1\end{array}$ \\
\hline$P S U_{n}(q)$ & $N_{1}=\frac{1}{d} G U_{n-1}(q)$ & $P_{m}=q^{m^{2}}:\left(\frac{1}{d} S L_{m}\left(q^{2}\right) \cdot(q-1)\right)$ & $\begin{array}{l}n=2 m, 2 \mid m \text { and } q \text { is even, } \\
d=(n, q+1)\end{array}$ \\
\hline$P \Omega_{2 m}^{+}(q)$ & $N_{1}=\Omega_{2 m-1}(q)$ & $\begin{array}{l}P_{m}=q^{m(m-1) / 2}: \frac{1}{d} G L_{m}(q) \cong \\
P_{m-1}\end{array}$ & $m$ is odd, $q$ is even, $d=(2, q-1)$ \\
\hline
\end{tabular}

Case 1. $G \cong P S L_{n}(q), n \geq 2$.

Then $|G|=\frac{q^{n(n-1) / 2}}{d} \prod_{i=2}^{n}\left(q^{i}-1\right)$, where $d=(n, q-1)$.

(1) $M={ }^{\wedge} G L_{a}\left(q^{b}\right) \cdot b$ and $N=P_{1}, a b=n$ and $b$ is a prime.

By [11, Proposition 4.3.6], $|M|=\frac{b}{d(q-1)} q^{n(a-1) / 2} \prod_{i=1}^{a}\left(q^{b i}-1\right)$ and then

$$
|G: M|=\frac{q^{n(n-a) / 2}}{b} \prod_{i=1, b \nmid i}^{n-1}\left(q^{i}-1\right) .
$$

By [11, Proposition 4.1.6], we have $N=P_{1} \cong q^{n-1}:\left(\frac{1}{d} G L_{n-1}(q)\right)$. Hence

$$
|N|=\frac{1}{d} q^{n(n-1) / 2} \prod_{i=1}^{n-1}\left(q^{i}-1\right)
$$

and $|G: N|=\frac{q^{n}-1}{q-1}$. By the hypothesis of coprimality, we get that $\left(q^{n}-1, q^{i}-1\right)=q-1$ for $b \nmid i$ and $i=2, \ldots, n$. It follows that $(n, i)=1$. This forces that $n=b^{s}$ and $a=b^{s-1}$. Take any $i$ such that $(n, i)=1$. We have that $\left(\frac{q^{n}-1}{q-1}, q^{i}-1\right)=(n, q-1)$ by Lemma 3.2. Since $(|G: M|,|G: N|)=1$, we get that $(n, q-1)=1$. In particular, $b \nmid(q-1)$.

(2) $M=P S p_{n}(q) . a$ and $N=P_{1}$ or $P_{n-1}$, where $a=(2, q-1)(n / 2, q-1) /(n, q-1)$ and $n \geq 4$ is even.

Then $|M|=a q^{n^{2} / 4} \prod_{i=1}^{n / 2}\left(q^{2 i}-1\right)$ and

$$
|G: M|=\frac{q^{n(n-2) / 4}}{a(n, q-1)} \frac{\left(q^{n-1}-1\right)\left(q^{n-2}-1\right) \cdots\left(q^{3}-1\right)}{\left(q^{n-2}-1\right)\left(q^{n-4}-1\right) \cdots\left(q^{4}-1\right)}=\frac{q^{n(n-2) / 4}}{a(n, q-1)} \prod_{i=2}^{n / 2}\left(q^{2 i-1}-1\right) .
$$

Clearly, $\left|P_{1}\right|=\left|P_{n-1}\right|$. Hence $|N|=\frac{1}{d} q^{n(n-1) / 2} \prod_{i=1}^{n-1}\left(q^{i}-1\right)$ and $|G: N|=\frac{q^{n}-1}{q-1}$. 
Since $(|G: M|,|G: N|)=1$, we have $(n, n-i)=1$ for every $i=1,3,5, \ldots, n-1$. Then $n$ is a power of 2 . Write $n=2^{s}$ with $s \geq 2$. By the same reason as in (1) of this case, we can get that $q-1$ is odd, in particular, $q$ is even.

(3) $M=P S p_{n}(q) \cdot a$ and $N=\operatorname{Stab}\left(V_{1} \oplus V_{n-1}\right)$, where $a=(2, q-1)(n / 2, q-1) /(n, q-1)$ and $n \geq 4$ is even.

Since $\left|P S p_{n}(q)\right|=\frac{q^{n^{2} / 4}}{(2, q-1)} \prod_{i=1}^{n / 2}\left(q^{2 i}-1\right)$, we have

$$
|G: M|=\frac{q^{n(n-2) / 4}}{a(n, q-1)}\left(q^{n-1}-1\right)\left(q^{n-3}-1\right)\left(q^{n-5}-1\right) \cdots\left(q^{3}-1\right) .
$$

By [11, Proposition 4.1.4], we have $N=\frac{1}{d} G L_{n-1}(q)$, so

$$
|N|=\frac{1}{d} q^{(q-1)(q-2) / 2} \prod_{i=1}^{n-1}\left(q^{i}-1\right)
$$

Consequently, $|G: N|=\frac{q^{n-1}\left(q^{n}-1\right)}{q-1}$. But in this case $(|G: M|,|G: N|) \neq 1$, a contradiction.

Case 2. $G \cong P S p_{2 m}(q)$ with $m \geq 2$.

Let $d=(2, q-1)$. Then $|G|=\frac{q^{m^{2}}}{d} \prod_{i=1}^{m}\left(q^{2 i}-1\right)$. By [11, Proposition 4.1.19], we have $P_{1}=q^{2 m-1}: \frac{1}{d}\left(P S p_{2(m-1)}(q) \times(q-1)\right)$ and $P_{m}=q^{m(m+1) / 2}: \frac{1}{d} G L_{m}(q)$. Thus, $\left|P_{m}\right|=\frac{1}{d} q^{m^{2}} \prod_{i=1}^{m}\left(q^{i}-1\right)$ and $\left|G: P_{m}\right|=\prod_{i=1}^{m}\left(q^{i}+1\right)$.

(1) $M \cong P S p_{2 a}\left(q^{b}\right) \cdot b$ and $N=P_{1}$.

Then $|M|=\frac{b}{d} q^{m a} \prod_{i=1}^{a}\left(q^{2 b i}-1\right)$ and $|G: M|=\frac{1}{b} q^{m(m-a)} \prod_{i=1, b \nmid i}^{a}\left(q^{2 i}-1\right)$. We also have that $|N|=\frac{1}{d^{2}} q^{m^{2}}(q-1) \prod_{i=1}^{m-1}\left(q^{2 i}-1\right)$ and $|G: N|=\frac{d\left(q^{2 m}-1\right)}{q-1}$. Lemma 3.2 implies that $\left(q^{2}-1\right) \mid\left(q^{2 m}-1, q^{2 i}-1\right)$. This gives $(|G: M|,|G: N|) \neq 1$, a contradiction.

(2) $M=S p_{2 a}\left(q^{b}\right) \cdot b$ and $N=O_{2 m}^{+}(q)$ or $O_{2 m}^{-}(q)$, where $q$ is even and $a b=m$ with $b$ a prime.

Then $|M|=b q^{m^{2} / b} \prod_{i=1}^{a}\left(q^{2 i}-1\right)$ and

$$
|N|=\frac{1}{2} q^{m(m-1)}\left(q^{m}-1\right) \prod_{i=1}^{m-1}\left(q^{2 i}-1\right), \text { or } \quad|N|=\frac{1}{2} q^{m(m-1)}\left(q^{m}+1\right) \prod_{i=1}^{m-1}\left(q^{2 i}-1\right) .
$$

Hence $|G: M|=\frac{1}{b} q^{m a(b-1)} \prod_{i=1, b \nmid i}^{a}\left(q^{2 i}-1\right)$ and $|G: N|=\frac{2}{d} q^{m}\left(q^{m}+1\right)$ or $\frac{2}{d} q^{m}\left(q^{m}-1\right)$. Clearly, both indexes are not coprime, a contradiction. 
(3). $M=O_{2 m}^{-}(q), N=P_{m}$ and $q$ is even.

Since $|M|=\frac{1}{2} q^{m(m-1)}\left(q^{m}+1\right) \prod_{i=1}^{m-1}\left(q^{2 i}-1\right)$ and $|N|=q^{m^{2}} \prod_{i=1}^{m}\left(q^{i}-1\right)$, it follows that $|G: M|=q^{m}\left(q^{m}-1\right)$ and $|G: N|=\prod_{i=1}^{m}\left(q^{i}+1\right)$.

Let $q=2^{t}$. Assume that $m t /(m t, i t)=m /(m, i)$ is even and $i t /(m t, i t)=i /(m, i)$ is odd, so $\left(2^{m t}-1,2^{t i}+1\right)=2^{(m t, i t)}+1$ by Lemma 3.3 , and we easily get a contradiction. Therefore, we can assume that there is no $i$ with $1 \leq i \leq m$ such that $m /(m, i)$ is even and $i /(m, i)$ is odd. If $m$ is even, we take $i=1$. Then $m /(m, i)$ is even and $i /(m, i)$ is odd, a contradiction. If $m$ is odd, then $\left(q^{m}-1, q^{i}+1\right)=1$ for every $1 \leq i \leq m$ by Lemma 3.3. It follows that if $G \cong P S p_{2 m}(q)$ with $m \geq 2$, then $M=O_{2 m}^{-}(q)$ and $\bar{N}=P_{m}$, with $q$ and $m$ even, are the desired subgroups.

(4) $M=S z(q)$ and $N=O_{4}^{+}(q)$, and $m=2, q=2^{f}, f \geq 3$ odd.

We have $|G|=q^{4}\left(q^{2}-1\right)\left(q^{4}-1\right),|M|=q^{2}\left(q^{2}+1\right)(q-1)$ and $|N|=\frac{1}{2} q^{2}\left(q^{2}-1\right)^{2}$. Hence $|G: M|=q^{2}(q+1)^{2}(q-1)$ and $|G: N|=2 q^{2}\left(q^{2}+1\right)$. Notice that $q^{2} \mid(|G: M|,|G: N|)$, which is a contradiction.

(5) $M=G_{2}(q)$ and $N=O_{6}^{+}(q), O_{6}^{-}(q), P_{1}$ or $N_{2}$. In addition, $m=3$ and $q$ even.

In this case $|G|=q^{9}\left(q^{2}-1\right)\left(q^{4}-1\right)\left(q^{6}-1\right), d=1$ and $|M|=q^{6}\left(q^{6}-1\right)\left(q^{2}-1\right)$. Thus $|G: M|=q^{3}\left(q^{4}-1\right)$. By [11, Proposition 4.1.3], we know that $N_{2}=P S p_{2}(q) \times P S p_{4}(q)$. Hence $|N|=\frac{1}{2} q^{6}\left(q^{2}-1\right)\left(q^{3}-1\right)\left(q^{4}-1\right), \frac{1}{2} q^{6}\left(q^{2}-1\right)\left(q^{3}+1\right)\left(q^{4}-1\right), q^{9}(q-1)\left(q^{2}-1\right)\left(q^{3}-1\right)$ or $q^{4}\left(q^{4}+q^{2}+1\right)$. It easily leads to a contradiction.

Case 3. $G=P S U_{n}(q), n \geq 3$ is even.

Let $d=(n, q+1)$ and $n=2 m$. Then $|G|=\frac{q^{\left(n^{2}-n\right) / 2}}{d} \prod_{i=2}^{n}\left(q^{i}-(-1)^{i}\right)$. By [11, Proposition 4.1.4], we have $P_{m}=q^{m^{2}}:\left(\frac{1}{d} S L_{m}\left(q^{2}\right) \cdot(q-1)\right)$ and $N_{1}=\frac{1}{d} G U_{n-1}(q)$.

(1) $M=N_{1}$ and $N=P_{m}$.

Then $|M|=\frac{(q-1)}{d} q^{2 m^{2}-m} \prod_{i=2}^{m}\left(q^{2 i}-1\right)$ and $|N|=\frac{q^{m(2 m-1)}}{d(q+1)} \prod_{i=1}^{m}\left(q^{2 i}-1\right)$. So $|G: M|=$ $q^{2 m-1}\left(q^{2 m}-1\right) /(q+1)$ and $|G: N|=\prod_{i=1}^{m}\left(q^{2 i-1}+1\right)$.

Assume that $m$ is even and write $n=2^{a} m_{1}$, where $a$ and $m_{1}$ are positive integers and $\left(2, m_{1}\right)=1$. It follows that $q^{m}-1=\left(q^{m_{1}}-1\right) \prod_{i=0}^{a-1}\left(q^{2^{i} m_{1}}+1\right)$. We have that $\frac{q^{m_{1}+1}}{q+1}$ is an integer. Notice that $\left(q^{m_{1}}-1, q^{2 i-1}+1\right)=(2, q+1)$ and $\left(q^{2^{j} m_{1}}+1, q^{2 i-1}+1\right)=(2, q+1)$ by Lemma 3.3. If $q$ is odd, we get $(|G: M|,|G: N|) \neq 1$, a contradiction. If $q$ is even, we obtain $\left(q^{2^{j} m_{1}}+1, q^{2 i-1}+1\right)=1$ and $\left(q^{m_{1}}-1, q^{2 i-1}+1\right)=1$ by Lemma 3.3. So we conclude that $(|G: M|,|G: N|)=1$. So in this case, the subgroups $M=N_{1}$ and $N=P_{m}$ are the desired subgroups. 
Assume now that $m$ is odd, so $\frac{q^{m}+1}{q+1}$ is an integer. As $\left(q^{m}-1, q^{2 i-1}+1\right)=q^{(m, 2 i-1)}+1$ by Lemma 3.3, we deduce that $(|G: M|,|G: N|) \neq 1$, a contradiction.

(2) $M=N_{1}$ and $N=P S p_{2 m}(q) . a, n=2 m, a=\frac{(2, q-1)(m, q+1)}{(n, q+1)}$.

We have $|G: M|=q^{2 m-1}\left(q^{2 m}-1\right) /(q+1)$. Since $|N|=\frac{a q^{m^{2}}}{(2, q-1)} \prod_{i=1}^{m}\left(q^{2 i}-1\right)$, we deduce that $q|| G: N \mid$, which is a contradiction.

Case 4. $G=\Omega_{2 m+1}(q), m \geq 3, q$ odd.

In this case, $|G|=\frac{1}{2} q^{m^{2}} \prod_{i=1}^{m}\left(q^{2 i}-1\right)$. By [11, Proposition 4.1.20], we know that $P_{m}=$ $q^{m(m-1) / 2}: \frac{1}{2} G L_{m}(q)$ and $P_{1}=q^{2 m-2}:\left(\frac{1}{2} G L_{1}(q) \times \Omega_{2 m-1}(q)\right) .2$. It follows that $\left|P_{m}\right|=$ $\frac{1}{2} q^{m(m-1)} \prod_{i=1}^{m}\left(q^{i}-1\right)$ and $\left|P_{1}\right|=\frac{1}{(2, q-1)} q^{m^{2}-1}(q-1) \prod_{i=1}^{m-1}\left(q^{2 i}-1\right)$. Hence

$$
\left|G: P_{m}\right|=q^{m} \prod_{i=1}^{m}\left(q^{i}+1\right)
$$

By [11, Proposition 4.1.6], we have $N_{1}^{-}=\Omega_{2 m}^{-}(q) .2$ and $\left|N_{1}^{-}\right|=q^{m^{2}-m}\left(q^{m}+1\right) \prod_{i=1}^{m-1}\left(q^{2 i}-1\right)$.

(1) $M=N_{1}^{-}$and $N=P_{m}$.

Hence $|G: M|=\frac{1}{2} q^{m}\left(q^{m}-1\right)$ and $|G: N|=q^{m} \prod_{i=1}^{m}\left(q^{i}+1\right)$. So we get that $q$ divides both indexes, a contradiction.

(2) $m=3, M=G_{2}(q)$ and $N=P_{1}, N_{1}^{+}, N_{1}^{-}$or $N_{2}^{-}$.

In this case $G=\Omega_{7}(q),|G|=\frac{1}{2} q^{9}\left(q^{6}-1\right)\left(q^{4}-1\right)\left(q^{2}-1\right)$ and $|M|=q^{6}\left(q^{6}-1\right)\left(q^{2}-1\right)$. Therefore, $|G: M|=\frac{1}{2} q^{3}\left(q^{4}-1\right)$.

By [11, Proposition 4.1.6], we have $N_{1}^{+}=\Omega_{6}^{+}(q) .2$ and $N_{2}^{-}=\left(\Omega_{2}^{\epsilon}(q) \times \Omega_{5}(q)\right) .2^{2}$, where $\epsilon= \pm 1$. Hence $\left|P_{1}\right|=\frac{1}{2} q^{8}(q-1)\left(q^{2}-1\right)\left(q^{4}-1\right),\left|N_{1}^{+}\right|=q^{6}\left(q^{3}-1\right)\left(q^{2}-1\right)\left(q^{4}-1\right)$, $\left|N_{1}^{-}\right|=q^{6}\left(q^{3}+1\right)\left(q^{2}-1\right)\left(q^{4}-1\right)$ and $\left|N_{2}^{-}\right|=q^{4}(q-\epsilon)\left(q^{2}-1\right)\left(q^{4}-1\right)$. It follows that $|G: N|=\frac{1}{(q-1)} q\left(q^{6}-1\right), \frac{1}{2} q^{3}\left(q^{3}+1\right), \frac{1}{2} q^{3}\left(q^{3}-1\right)$ or $\frac{1}{2} q \frac{\left(q^{6}-1\right)}{q-\epsilon}$. It is easy to see that this leads to $(|G: M|,|G: N|) \neq 1$, a contradiction.

(3) $m=3, M=G_{2}(q)$ and $N=N_{2}^{+}$.

By the same reason as in (2), this case can be ruled out.

(4) $m=6, M=P S p_{6}(q) \cdot a(a=1,2)$ and $N=N_{1}^{-}$.

In this case $|G|=\left|\Omega_{13}(q)\right|=\frac{1}{2} q^{36}\left(q^{12}-1\right)\left(q^{10}-1\right) \cdots\left(q^{2}-1\right)$

$$
|M|=\frac{1}{2} q^{9}\left(q^{2}-1\right)\left(q^{4}-1\right)\left(q^{6}-1\right) a \quad \text { and } \quad|N|=q^{30}\left(q^{6}+1\right) \prod_{i=1}^{5}\left(q^{2 i}-1\right) .
$$


Hence $|G: M|=q^{27}\left(q^{12}-1\right)\left(q^{10}-1\right)\left(q^{8}-1\right) / a$ and $|G: N|=\frac{1}{2} q^{6}\left(q^{6}-1\right)$. So we get that $(|G: M|,|G: N|) \neq 1$, a contradiction.

(5) $m=12, M=F_{4}(q)$ and $N=N_{1}^{-}$.

Note that $|G: N|=\frac{1}{2} q^{m}\left(q^{m}-1\right)$. By the same argument as in (2), we obtain a contradiction.

Case 5. $G=P \Omega_{2 m}^{-}(q), m \geq 4$.

(1) $M=P_{1}$ and $N=\wedge G U_{m}(q), m$ odd.

In this case $|G|=\frac{1}{\left(4, q^{m}+1\right)} q^{m(m-1)}\left(q^{m}+1\right) \prod_{i=1}^{m-1}\left(q^{2 i}-1\right)$. By [11, Proposition 4.1.6], we have

$$
\left|P_{1}\right|=\frac{1}{d\left(4, q^{m-1}+1\right)} q^{(m-1)(m-2)}(q-1)\left(q^{m-1}+1\right) \prod_{i=1}^{m-2}\left(q^{2 i}-1\right),
$$

where $d=1$ or 2 . It follows that

$$
|G: M|=\frac{d\left(4, q^{m-1}+1\right)}{\left(4, q^{m}+1\right)} q^{2 m-2}\left(q^{m}+1\right)\left(q^{m-1}-1\right) /(q-1) .
$$

By [11, Proposition 4.1.18], we have that $N \cong \frac{q+1}{a} \cdot U_{m}(q) \cdot[(q+1, m)]$, where $a=(q+1,4)$. Hence $|N|=\frac{(q+1)}{a} q^{\left(m^{2}-m\right) / 2} \prod_{i=1}^{m}\left(q^{i}-(-1)^{i}\right)$ and $|G: N|=\frac{a\left(q^{m-1}+1\right)}{(q+1)} q^{\left(m^{2}-m\right) / 2} \prod_{i=1}^{m-2} \frac{q^{2 i}-1}{q^{i}-(-1)^{i}}$. So we get that $q \mid(|G: M|,|G: N|)$, a contradiction.

(2) $M=N_{1}$ and $N={ }^{\wedge} G U_{m}(q), m$ odd.

By [11, Proposition 4.1.6], we have $M=\Omega_{2 m-1}(q)$ and thus

$$
|G: M|=\frac{1}{\left(4, q^{m}+1\right)} q^{m-1}\left(q^{m}+1\right) .
$$

Arguing as in (2), we obtain

$$
|G: N|=\frac{a}{(q+1)} q^{\left(m^{2}-m\right) / 2}\left(q^{m-1}+1\right) \prod_{i=1}^{m-2} \frac{q^{2 i}-1}{q^{i}-(-1)^{i}},
$$

where $a=(4, q+1)$. This implies that $q \mid(|G: M|,|G: N|)$, a contradiction.

Case 6. $G=P \Omega_{2 m}^{+}(q), m \geq 5$.

In this case, we have $|G|=\frac{1}{\left(4, q^{m}-1\right)} q^{m(m-1)}\left(q^{m}-1\right) \prod_{i=1}^{m-1}\left(q^{2 i}-1\right)$ and $d=(2, q-1)$. Clearly, $\left|P_{m}\right|=\left|P_{m-1}\right|$. By [11, Proposition 4.1.20], we know that $P_{m}=q^{m(m-1) / 2}$ : $\frac{1}{d} G L_{m}(q)$. Hence $\left|P_{m}\right|=\frac{q^{m(m-1)}}{d} \prod_{i=1}^{m}\left(q^{i}-1\right)$ and $\left|G: P_{m}\right|=\frac{d}{\left(4, q^{m}-1\right)} \prod_{i=1}^{m-1}\left(q^{i}+1\right)$.

(1) $M=N_{1}$ and $N=P_{m}$ or $P_{m-1}$. 
By [11, Proposition 4.1.6], $M=\Omega_{2 m-1}(q)$ and thus $|G: M|=\frac{2}{\left(4, q^{m}-1\right)} q^{m-1}\left(q^{m}-1\right)$. Note that $|G: N|=\frac{d}{\left(4, q^{m}-1\right)} \prod_{i=1}^{m-1}\left(q^{i}+1\right)$. Assume that $m$ is even. We can get that $\left(q^{m / 2}+1\right) \mid(|G: M|,|G: N|)$, a contradiction. So we assume that $m$ is odd.

If $q \equiv-1(4)$, then $\frac{2}{\left(4, q^{m}-1\right)}=1$ and $\frac{d}{\left(4, q^{m}-1\right)}=1$. By Lemma 3.3, $\left(q^{m}-1, q^{i}+1\right)=2$ for every $1 \leq i \leq m-1$. So we get that $(|G: M|,|G: N|) \neq 1$, a contradiction. If $q \equiv 1(4)$, then $4 \mid\left(q^{m}-1\right), \frac{2}{\left(4, q^{m}-1\right)}=\frac{1}{2}$ and $\frac{d}{\left(4, q^{m}-1\right)}=\frac{1}{2}$. By Lemma 3.3, we have $\left(q^{m}-1, q^{i}+1\right)=2$ for every $1 \leq i \leq m-1$. Therefore, $2 \mid(|G: M|,|G: N|)$, a contradiction.

If $q$ is even, then $\left(q^{m}-1, q^{i}+1\right)=1$ for every $1 \leq i \leq m-1$ by Lemma 3.3. Furthermore, $(|G: M|,|G: N|)=1$. We conclude that $M$ and $N$ are the desired subgroups.

(2) $M=N_{1}$ and $N={ }^{\wedge} G U_{m}(q) .2$, where $m$ is even.

By [11, Proposition 4.1.18], we have that $|N|=\frac{2 b}{a} q^{\left(m^{2}-m\right) / 2} \prod_{i=1}^{m}\left(q^{i}-(-1)^{i}\right)$, where $a=(q+1,2)$ and $b=1$ if $q$ is odd, $b=2$ if $q$ is even. Hence

$$
|G: N|=\frac{a}{2 b\left(4, q^{m}-1\right)} q^{\left(m^{2}-m\right) / 2} \prod_{i=1}^{m-1}\left(q^{i}+(-1)^{i}\right) .
$$

So we get that $q \mid(|G: M|,|G: N|)$, a contradiction.

(3) $M=N_{1}$ and $N=\left(P S p_{2}(q) \otimes P S p_{m}(q)\right) . a$, where $m$ is even, $q>2$ and $a=$ $(2, m / 2, q-1)$.

By [11, Proposition 4.1.12], we have $|N|=\frac{a c\left(q^{2}-1\right)}{2(2, q-1)} q^{\left(m^{2} / 4+1\right)} \prod_{i=1}^{m / 2}\left(q^{2 i}-1\right)$, where $c=1$ or 2. Hence $|G: N|=\frac{2(2, q-1)\left(q^{m}-1\right)}{a c\left(4, q^{m}-1\right)\left(q^{2}-1\right)} q^{\left(3 m^{2} / 4-m-1\right)} \prod_{i=m / 2+1}^{m-1}\left(q^{2 i}-1\right)$. By (1) of Case 6, we have $|G: M|=\frac{1}{\left(4, q^{m}-1\right)} q^{m-1}\left(q^{m}-1\right)$. So we conclude that $q \mid(|G: M|,|G: N|)$, a contradiction.

(4) $M=N_{2}^{-}$and $N=P_{m}$ or $P_{m-1}$.

By $\left[11\right.$, Proposition 4.1.6], we have $|M|=\frac{d}{(2, q-1)} q^{(m-1)(m-2)}(q \pm 1)\left(q^{m-1} \pm 1\right) \prod_{i=1}^{m-2}\left(q^{2 i}-1\right)$, where $d=1$ or 2 , or $|M|=\frac{8}{\left(4, q^{m-1}-\epsilon 1\right)} q^{(m-1)(m-2)}(q-\epsilon 1) \prod_{i=1}^{m-2}\left(q^{2 i}-1\right)$, where $\epsilon= \pm 1$. Hence

$$
\begin{gathered}
|G: M|=\frac{(2, q-1)}{d\left(4, q^{m}-1\right)} q^{2 m-2}\left(q^{m-1} \mp 1\right)\left(q^{m}-1\right) /(q \pm 1), \text { or } \\
|G: M|=\frac{\left(4, q^{m-1} \pm 1\right)}{8\left(4, q^{m}-1\right)} q^{2 m-2}\left(q^{m-1} \mp 1\right)\left(q^{m-1} \pm 1\right)\left(q^{m}-1\right) /(q \pm 1) .
\end{gathered}
$$

Note that $\left|P_{m}\right|=\left|P_{m-1}\right|$ and $|G: N|=\frac{(2, q-1)\left(q^{2}-1\right)}{\left(4, q^{m}-1\right)} \prod_{i=1}^{m-1}\left(q^{i}+1\right)$. Assume first that $\left(q^{m-1}-1\right)|| G: M \mid$. If $m$ is even, we get that $\frac{1}{2(q+1)} q^{2 m-2}\left(q^{m-1}-1\right)$ and $\frac{(2, q-1)\left(q^{2}-1\right)}{\left(4, q^{m}-1\right)}$ are 
integers. Since $\left(q^{m / 2}+1\right) \mid\left(q^{m}-1, \prod_{i=1}^{m-1}\left(q^{i}+1\right)\right)$, we have that $\left(q^{m / 2}+1\right) \mid(|G: M|,|G: N|)$, a contradiction. If $m$ is odd, we also can get that $\left(q^{(m-1) / 2}+1\right) \mid\left(q^{m-1}-1, \prod_{i=1}^{m-1}\left(q^{i}+1\right)\right)$, again a contradiction.

Assume now that $\left(q^{m-1}+1\right)|| G: M \mid$. Clearly, $\left(q^{m-1}+1\right) \mid(|G: M|,|G: N|)$, a contradiction.

(5) $M=P_{1}$ and $N={ }^{\wedge} G U_{m}(q) .2$, where $m$ is even.

By [11, Proposition 4.1.20], we get $\left|P_{1}\right|=\frac{1}{2(2, q-1)} q^{m^{2}-m}\left(q^{m-1}-1\right)(q-1) \prod_{i=1}^{m-2}\left(q^{2 i}-1\right)$. Hence $|G: M|=\frac{2(2, q-1)}{\left(4, q^{m}-1\right)(q-1)}\left(q^{m}-1\right)\left(q^{m-1}+1\right)$.

By (2), we have $|G: N|=\frac{(4, q+1)}{2 b\left(4, q^{m}-1\right)} q^{\left(m^{2}-m\right) / 2} \prod_{i=1}^{m-1}\left(q^{i}+(-1)^{i}\right)$. If $m / 2$ is even, then $\left(q^{m / 2}+1\right) \mid\left(q^{m}-1, \prod_{i=1}^{m-1}\left(q^{i}+(-1)^{i}\right)\right)$. If If $m / 2$ is odd, we have that $\left(q^{m / 2}-1\right)$ divides $\left(q^{m}-1, \prod_{i=1}^{m-1}\left(q^{i}+(-1)^{i}\right)\right)$. This yields a contradiction.

(6) $M=N_{1}$ and $N={ }^{\wedge} G L_{m}(q) \cdot 2$.

By [11, Proposition 4.1.6], we have $|M|=\Omega_{2 m-1}(q)$ and thus $|G: M|=\frac{2}{\left(4, q^{m}-1\right)} q^{m-1}\left(q^{m}-\right.$ 1). By [11, Proposition 4.1.7],

$$
\begin{aligned}
|N|= & (2, m) q^{\left(m^{2}-m\right) / 2} \prod_{i=1}^{m}\left(q^{i}-1\right) \text { or } \\
& \frac{1}{d} q^{\left(m^{2}-m\right) / 2} \prod_{i=1}^{m}\left(q^{i}-1\right),
\end{aligned}
$$

where $d=2$ or 4 . Hence

$$
\begin{aligned}
|G: N|= & \frac{1}{\left(4, q^{m}-1\right)(2, m)} q^{\left(m^{2}-m\right) / 2} \prod_{i=1}^{m-1}\left(q^{i}+1\right), \text { or } \\
& \frac{d}{\left(4, q^{m}-1\right)} q^{\left(m^{2}-m\right) / 2} \prod_{i=1}^{m-1}\left(q^{i}-1\right) .
\end{aligned}
$$

This shows that $q \mid(|G: M|,|G: N|)$, a contradiction. 


\section{Proof of Theorem B}

We will prove first the solvability in Theorem B. We use $\pi(G)$ to denote the set of primes dividing $|G|$. If $n$ is an integer and $p$ is a prime, $n_{p}$ will denote the $p$-part of $n$. We will use the following standard lemma to deal with the action on direct product of simple groups.

Lemma 4.1. $[12,8.1 .6$ (a)] Suppose that a finite group $A$ acts on a finite group $G$ which allows a direct decomposition $G=H_{1} \times \cdots \times H_{n}$, that is $A$-invariant under $A$, i.e., $H_{i}^{a} \in\left\{H_{1}, \ldots, H_{n}\right\}$ for all $a \in A$ and all $i \in\{1, \ldots, n\}$. Assume further that $A$ acts transitively on $\left\{H_{1}, \ldots, H_{n}\right\}$. Let $H \in\left\{H_{1}, \ldots, H_{n}\right\}$ and let $B=\mathbf{N}_{A}(H)$ and let $T a$ transversal of $B$ in $A$. Then

(a) $\mathbf{C}_{G}(A)=\left\{\prod_{t \in T} c^{t} \mid c \in \mathbf{C}_{H}(B)\right\} \cong \mathbf{C}_{H}(B)$.

(b) if $B$ acts trivially on $H$ and $P \leq H$ such that $\left\langle P^{H}\right\rangle=H$, then

$$
G=\left\langle\mathbf{C}_{G}(A), \prod_{t \in T} P^{t}\right\rangle
$$

Theorem 4.2. Suppose that a finite group $A$ acts coprimely on a finite group $G$. If all maximal $A$-invariant subgroups of $G$ lie in two $\mathbf{C}_{G}(A)$-orbits, then $G$ is solvable

Proof. We argue by minimal counterexample. Let $C:=\mathbf{C}_{G}(A)$ and choose $M$ and $N$ to be two maximal $A$-invariant subgroups of $G$ that are not $C$-conjugate. Of course, we can assume that $G$ is not a prime power order group. First we show that $(|G: M|,|G: N|)=$ 1. Assume that there is a prime $p$ dividing $(|G: M|,|G: N|)$. Let $P \in \operatorname{Syl}_{p}^{A}(G)$ and let $U$ be a maximal $A$-invariant subgroup of $G$ such that $P \leq U$. Clearly, $U$ cannot be conjugate to $M$ or $N$, which gives a contradiction. Thus, $(|G: M|,|G: N|)=1$, and in particular, we get $G=M N$. We continue the proof with several steps.

Step 1. $G$ does not have any non-trivial proper $A$-invariant normal subgroup, and hence $G=S_{1} \times \ldots \times S_{n}$ with $S_{i}$ non-abelian simple isomorphic groups.

Suppose that $K<G$ is a minimal $A$-invariant normal subgroup of $G$. By minimality of $G$ (and Theorem A), it is obvious that $G$ must have trivial Fitting subgroup. Also, we can assume without loss that $K \leq N$. Let $p \in \pi(K)$ and $K_{p} \in \operatorname{Syl}_{p}^{A}(K)$. Then the Frattini argument gives $\mathbf{N}_{G}\left(K_{p}\right) K=G$, so $\mathbf{N}_{G}\left(K_{p}\right) \not \leq N^{c}$ for all $c \in C$. Now $\mathbf{N}_{G}\left(K_{p}\right)$ is $A$-invariant and proper in $G$, because $\mathbf{F}(G)=1$. As a consequence, $\mathbf{N}_{G}\left(K_{p}\right)^{c} \leq M$, for some $c \in C$. Since $K_{p}^{c}$ is also an $A$-invariant Sylow $p$-subgroup of $K$, by the arbitrariness of $p$ we deduce that $K \leq M$, which is impossible again by the Frattini argument. Therefore, $K=G$, and the step follows.

Step 2. $G$ is a simple group of Lie type.

Assume that $n \geq 1$ and write $S:=S_{1}$. By step 1 we know that $A$ acts transitively on the $S_{i}$. Let $B=\mathbf{N}_{A}(S)$ and $T$ a transversal of $B$ in $A$. As $B$ acts on $S$, by minimal counterexample, we can assume that $S$ has at least three maximal $B$-invariant subgroups, say $M_{1}, M_{2}$ and $M_{3}$, lying in different $\mathbf{C}_{S}(B)$-orbits. We certainly have that $\prod_{t \in T} M_{i}^{t}$ for $i=1,2,3$ are $A$-invariant subgroups of $G$, and there is no loss to assume that two of them, say $\prod_{t \in T} M_{1}^{t}$ and $\prod_{t \in T} M_{2}^{t}$ are respectively contained in two $C$-conjugate maximal $A$ invariant subgroups of $G$, say $M$ and $M^{c}$, with $c \in C$. It certainly follows that $M_{1} \subseteq M \cap S$ 
and $M_{2} \subseteq M^{c} \cap S$. Now, since $S \unlhd G$ then $M^{c} \cap S=(M \cap S)^{c}$, and by applying Lemma 4.1(a), $c$ can be assumed to belong to $\mathbf{C}_{S}(B)$. Hence we can assert that $M \cap S$ and $M^{c} \cap S$ are $B$-invariant subgroups of $S$. But the maximality of $M_{1}$ and $M_{2}$ implies then that $M_{1}=M \cap S$ and $M_{2}=M^{c} \cap S=(M \cap S)^{c}$, that is, $M_{1}$ and $M_{2}$ are conjugate in $\mathbf{C}_{S}(B)$, contradicting or assumption. This proves that $n=1$. Furthermore, we note that we can assume $C<G$, otherwise the action is trivial and our theorem is just the original version of Adnan, as pointed out in the Introduction. Now the fact that the alternating and the sporadic simple groups do not admit non-trivial coprime action allows to conclude that $S$ is simple of Lie type.

Step 3. Contradiction.

By minimal counterexample we can assume that $A$ acts faithfully on $G$, otherwise the action of $A / \mathbf{C}_{A}(G)$ on $G$ leads to a contradiction. Hence $A$ can be assumed to be (up to conjugation) an automorphism group of the underlying field of $G$, which has order, say $q=p^{f}$, for some prime $p$. In particular, $|A|$ must divide $f$, and since $(|G|,|A|)=1$ then $|A|$ is odd. As a consequence $f$ cannot be a power of 2. Thus, we can apply Theorem 3.4, so $(G, M, N)$ can be assumed to be one of the triples appearing in Table 1 . If one of $M$ and $N$ is not $A$-invariant, there will be a contradiction. Consequently, we may assume that both $M$ and $N$ are $A$-invariant, and certainly $M \nsubseteq N$. Let $A_{0}$ be a subgroup of $A$ of order $r$, where $r$ is a prime and $q=q_{0}^{r}$. Since $|A|$ is odd, we get that $r$ is odd too. Write $G=G(q)$. Then $G_{0}:=\mathbf{C}_{G}\left(A_{0}\right)=G\left(q_{0}\right)$, that is, $C$ is the same Lie type group as $G$ but defined over the field of $q_{0}$ elements. Since $A$ is a field automorphism group of $G$, we have that $A$ is cyclic, and as a result $G_{0}$ is $A$-invariant. Now we discuss the different possibilities according to Table 1 .

Case 1. $G=\operatorname{PSL}_{n}(q)$.

In this case, $G_{0}=\operatorname{PSL}_{n}\left(q_{0}\right)$. By Table 1 , we have $d=(n, q-1)=1$. So, by $[11$, Proposition 4.5.3], $G_{0}$ is a maximal $A$-invariant subgroup of $G$, which is not isomorphic to $M$ or $N$, a contradiction.

Similarly, we can rule out the cases $G=\operatorname{PSp}_{2 m}(q)$ and $G=\mathrm{P}_{2 m}^{+}(q)$.

Case 2. $G=\operatorname{PSU}_{n}(q)$.

In this case, $G_{0}=\operatorname{PSU}_{n}\left(q_{0}\right)$. Note that $G_{0}$ is $A$-invariant and all maximal $A$-invariant subgroups of $G$ lie in two $C$-orbits, so we have $G_{0} \leq M^{c}$ or $G_{0} \leq N^{c}$ for some $c \in C$. Without loss of generality, we may assume that $G_{0} \leq M$ or $G_{0} \leq N$. Assume first that $G_{0} \leq M$. Since $M=\frac{1}{d} G U_{n-1}(q)$ is $A$-invariant, $\mathbf{C}_{M}\left(A_{0}\right)=\frac{1}{d} G U_{n-1}\left(q_{0}\right)$ is also $A$-invariant. Thus we get $G_{0}=\frac{1}{d} G U_{n-1}\left(q_{0}\right)$, a contradiction. Assume then $G_{0} \leq N$. By [11, Proposition 4.5.3], it follows that $\mathbf{N}_{G}\left(G_{0}\right)$ is a maximal $A$-invariant subgroup of $G$. Hence $\mathbf{N}_{G}\left(G_{0}\right)=N=q^{m^{2}}:\left(\frac{1}{d} \mathrm{SL}_{m}\left(q^{2}\right) \cdot(q-1)\right)$. By comparing the orders of $\mathbf{N}_{G}\left(G_{0}\right)$ and $N$, we find the final contradiction.

Once we have proved the solvability in Theorem B, we address the structure part, which provides a more detailed description of those groups having exactly two orbits of maximal invariant subgroups. 
Theorem 4.3. Suppose that a group $A$ acts coprimely on a group $G$. If all maximal $A$-invariant subgroups of $G$ lie in two $\mathbf{C}_{G}(A)$-orbits, then one of the following conditions holds:

(1) $G$ is a prime power order group.

(2) $G=P Q$, where $P \in \operatorname{Syl}_{p}^{A}(G)$ and $Q \in \operatorname{Syl}_{q}^{A}(G)$, and $P \unlhd G$. In addition, $P / \Phi(P)$ is $Q A$-irreducible.

Proof. Write $C:=\mathbf{C}_{G}(A)$. Let $M$ and $N$ be two maximal $A$-invariant subgroups of $G$ that are not $C$-conjugate. By the above theorem $G$ is solvable, and by Lemma 2.1 we know that $|G: M|$ and $|G: N|$ are prime powers, say $p^{a}=|G: M|$ and $q^{b}=|G: N|$. Also, we will assume that $G$ has not prime power order, otherwise we get case (1). An earlier argument in the above theorem shows that $(|G: M|,|G: N|)=1$, which means that $p \neq q$. Next we prove that $G=P Q$, where $P \in \operatorname{Syl}_{p}^{A}(G)$ and $Q \in \operatorname{Syl}_{q}^{A}(G)$. Suppose on the contrary that $|\pi(G)| \geq 3$. The solvability of $G$ and Glauberman's Lemma (for instance [12, Theorem 6.2.2]) guarantee that $G$ has an $A$-invariant Hall $\{p, q\}$-subgroup, say $H$, and then we take a maximal $A$-invariant subgroup $W$ of $G$ such that $H \leq W$. Clearly, $W$ is not $C$-conjugate to $M$ or $N$, again a contradiction. We conclude that $|\pi(G)|=2$. For the following, we assume that $\pi(G)=\{p, q\}$, and if $P \in \operatorname{Syl}_{p}^{A}(G)$ and $Q \in \operatorname{Syl}_{q}^{A}(G)$, then we can write $G=P Q$, as wanted.

Let $S$ be a maximal $A$-invariant normal subgroup of $G$. The hypotheses imply that $S \leq M$ or $S \leq N$. Without loss of generality, we assume $S \leq M$. Since $G$ is solvable, by Lemma 2.2, we have that $|G: S|$ is a prime power. Likewise, without loss, we may assume that $|G: S|=q^{b}$, and thus $P \leq S$. By the Frattini argument, we have $G=\mathbf{N}_{G}(P) S$. Clearly, $\mathbf{N}_{G}(P)$ is $A$-invariant and $\mathbf{N}_{G}(P) \not \leq M^{c}$ for every $c \in C$. If $\mathbf{N}_{G}(P)<G$, then there is some $A$-invariant maximal subgroup $U$ of $G$ such that $\mathbf{N}_{G}(P) \leq U$. However, $U$ cannot be $C$-conjugate to $M$ or $N$, and this is a contradiction. Therefore $\mathbf{N}_{G}(P)=G$, that is, $P \unlhd G$ and the first part of (2) is proved.

To prove the last assertion of (2), we note that $Q A$ acts coprimely on $P / \Phi(P)$. If the action is not irreducible, Masche's Theorem $([12,8.4 .6])$ gives $P=H K$ for some $Q A$-invariant subgroups $H$ and $K$ such that $\Phi(P)<H, K<P$. Then $H Q$ and $K Q$ are $A$-invariant subgroups and not contained in $M$, because $P \leq M$ by the above paragraph. Since $H, K \unlhd G$, this yields $H, K \leq N$, hence $P \leq N$, which provides a contradiction.

We end this section with some examples illustrating that all the cases appearing in Theorems A and B occur and that the bound 2 in Theorem B is the best possible.

Example 1. Aside from cyclic $p$-groups acted on by coprime automorphisms, which always satisfy the hypothesis of Theorem A, the easiest example is the group $G=Q_{8}$ which has an automorphism of order 3 acting non-trivially. The only maximal invariant subgroup of $G$ is $\mathbf{Z}(G)=\Phi(G)$. A more general example is any extraspecial $p$-group $P$ of order $p^{2 n+1}$ with exponent $p$. It is possible to find a coprime automorphism of $P$, whose order divides $p^{n}+1$, acting trivially on $\mathbf{Z}(P)=\Phi(P)$ and irreducibly on $P / \mathbf{Z}(P)$ (see [17] for instance).

Example 2. We show that case (1) of Theorem B can happen, that is, there exists a finite $p$-group $P$ acted on by a group $A$ of coprime order such that $P$ has exactly two 
$\mathrm{C}_{P}(A)$-orbits of maximal $A$-invariant subgroups. Let $P$ be the extraspecial group of order 27 and exponent 3 , which has the following presentation

$$
P=\left\langle x, y, z \mid x^{3}=y^{3}=z^{3}=1,[x, z]=1,[y, z]=1, y x=x y z\right\rangle .
$$

We consider $a \in \operatorname{Aut}(P)$ defined by $x^{a}=y, z^{a}=z^{2}$ and $y^{a}=x$. So $o(a)=2, A=\langle a\rangle$ acts coprimely on $P$ and $C:=\mathbf{C}_{P}(A)=\left\langle x y z^{2}\right\rangle$.

Every maximal subgroup of $P$ contains $\mathbf{Z}(P)=\Phi(P)=\langle z\rangle$ and thus, there exists a one to one correspondence between the maximal subgroups of $P$ and the maximal subgroups of $P / \Phi(P) \cong C_{3} \times C_{3}$. This provides the only 4 maximal subgroups in $P$, which are exactly: $M_{1}=\langle x\rangle \times\langle z\rangle, M_{2}=\langle y\rangle \times\langle z\rangle, M_{3}=\langle x y\rangle \times\langle z\rangle$ and $M_{4}=\left\langle x^{2} y\right\rangle \times\langle z\rangle$. Now $A$ acts on the set of these four subgroups by permuting $M_{1}$ and $M_{2}$ and fixing $M_{3}$ and $M_{4}$. In particular, $M_{3}$ and $M_{4}$ are maximal $A$-invariant subgroups of $P$ and form two different $C$-orbits. There is not any other maximal $A$-invariant subgroup in $P$.

Example 3. The bound 2 for the number of orbits in Theorem B is the best possible even when the action is non-trivial. For example, let $G=\operatorname{PSL}(2,32)$ and let $a$ be the field automorphism of $\operatorname{GF}\left(2^{5}\right)$ of order 5 . Set $A=\langle a\rangle$. It follows that $C:=\mathbf{C}_{G}(A) \cong$ $\operatorname{PSL}(2,2)$. We know that $G$ has exactly three conjugacy classes of maximal subgroups (see for instance page 29 of [6]). Moreover, $A$ acts on each of these classes since the subgroups are normalizers of Sylow subgroups. By Glauberman's Lemma we can take from each class one $A$-invariant subgroup, and we obtain three maximal $A$-invariant subgroups lying in three different $C$-orbits.

Open problem. As we have pointed out in the Introduction, Belonogov proved that if a non-solvable group $G$ has exactly three conjugacy classes of maximal subgroups, then $G / \Phi(G)$ is isomorphic to $\operatorname{PSL}(2,7)$ or $\operatorname{PSL}\left(2,2^{p}\right)$ with $p$ a prime. Our question is: Is it possible to classify all non-abelian simple finite groups acted on by a coprime automorphism group and having exactly three orbits of maximal invariant subgroups under the action of the fixed point subgroup?

\section{Proof of Theorem C}

To address the case of one orbit of non-nilpotent invariant maximal subgroups in Theorem $\mathrm{C}$, we only use some results obtained by the authors in [4]. However, we remark that they are based on the CFSG too. We state here these results, the first of which extends Schmidt's theorem on minimal non-nilpotent groups.

Theorem 5.1. [4, Theorem A] Let $G$ and $A$ be finite groups of coprime orders and assume that $A$ acts on $G$ by automorphisms. If every maximal $A$-invariant subgroup of $G$ is nilpotent but $G$ is not, then $G$ is solvable and $|G|=p^{a} q^{b}$ for two distinct primes $p$ and $q$, and $G$ has a normal A-invariant Sylow subgroup.

Theorem 5.2. [4, Theorem D] Suppose that a finite group A acts coprimely on a finite group $G$ and let $p$ be a prime divisor of $|G|$. If the indices of all non-nilpotent maximal $A$-invariant subgroups of $G$ are powers of $p$, then $G$ is solvable. 
Lemma 5.3. [4, Lemma 5.3] Let $G$ be a finite group with $\mathbf{F}(G)=1$ and $G^{\prime}=G$ and suppose that a group $A$ acts coprimely on $G$. If $M$ is a nilpotent maximal A-invariant subgroup of $G$, then $M$ is a Sylow 2-subgroup of $G$.

Theorem 5.4. Suppose that a finite group $A$ acts coprimely on a finite group $G$. If all non-nilpotent maximal A-invariant subgroups of $G$ have the same index, then $G$ is solvable.

Proof. We induct on the order of $G$. If all maximal $A$-invariant subgroups of $G$ are nilpotent, then by Theorem 5.1, $G$ is solvable, so we can assume that $G$ has at least one non-nilpotent maximal $A$-invariant subgroup. Furthermore, we claim that $G$ must posses at least one nilpotent maximal $A$-invariant subgroup. Otherwise, all maximal $A$-invariant subgroups of $G$ would have the same index. Then, for every prime divisor of $G$ we may consider an $A$-invariant Sylow $p$-subgroup and take some maximal $A$-invariant subgroup containing it. All these subgroups have then the same index, what easily leads $G$ to have prime power order, and the theorem is proved. Thus the claim is proved.

Suppose now that $G$ has no proper non-trivial $A$-invariant normal subgroup and that $G$ is not solvable. We seek contradiction. Then we may write that $G=S_{1} \times \ldots \times S_{n}$ with $S_{i}$ non-abelian simple and isomorphic. For every prime $p \neq 2$, we take $P \in \operatorname{Syl}_{p}^{A}(G)$. It is clear that there exists a maximal $A$-invariant subgroup $H_{p}$ of $G$ containing $P$. As $G$ satisfies the hypotheses of Lemma 5.3 we get that $H_{p}$ cannot be nilpotent. Furthermore, $\left|G: H_{p}\right|$ is certainly a $p^{\prime}$-number for every $p$, and this fact joint with the hypotheses imply that $\left|G: H_{p}\right|$ is a power of 2 . Theorem 5.2 applies, so $G$ is solvable, a contradiction.

By the above paragraph, there exists a proper $A$-invariant normal subgroup $1<N$ of $G$, so $G / N$ is solvable, by induction. Also, $N$ can be assumed to be non-solvable or the proof would be finished. As we have seen above, we can take a nilpotent maximal $A$-invariant subgroup $H$ of $G$. Then $H N=G$ and $G / N \cong H / H \cap N$ is nilpotent. In particular, every maximal $A$-invariant subgroup of $G / N$ provides an $A$-invariant maximal subgroup of $G$ containing $N$. Since $N$ is not nilpotent, such subgroups are not nilpotent, and hence have the same index in $G$. This forces $G / N$ to be a $p$-group for some prime $p$. By hypothesis, all non-nilpotent maximal $A$-invariant subgroups have a $p$-power index, and again by Theorem 5.2, we have that $G$ is solvable. This completes the proof.

Corollary 5.5. Suppose that a finite group $A$ acts coprimely on a finite group $G$. If all non-nilpotent maximal $A$-invariant subgroups of $G$ are $\mathbf{C}_{G}(A)$-conjugate, then $G$ is solvable.

Proof. If there is only one $\mathbf{C}_{G}(A)$-orbit of such subgroups, then all these subgroups have the same index, and we can apply Theorem 5.4.

The case of two orbits in Theorem $\mathrm{C}$ requires a more detailed analysis on simple groups. Next we present some results that will be necessary for proving it. The first one is a deep classification due to Kondriat'ev.

Lemma 5.6. [9, Corollary] Let $G$ be a non-abelian simple group of Lie type over a field of characteristic $r$. Let $P$ be a Sylow 2-subgroup of $G$. If $\mathbf{N}_{G}(P)=P$, then $r$ is odd and $G$ is isomorphic to one of the following groups: 
$A_{1}(q) \cong \operatorname{PSL}(2, q)$, with $q \equiv \pm 1(\bmod 8) ; B_{n}(q) ; C_{n}(q) \cong \operatorname{PSp}(2 n, q) \cong S_{2 n}(q)$ with $n=1$ or $n \geq 2$ and $q \equiv \pm 1(\bmod 8) ; D_{n}(q) \cong \mathrm{P} \Omega^{+}(2 n, q)(n \geq 4) ; E_{7}(q), E_{8}(q), F_{4}(q)$, $G_{2}(q),{ }^{2} D_{n}(q) \cong \mathrm{P} \Omega^{-}\left(2 n, q^{2}\right)(n \geq 4)$ or ${ }^{3} D_{4}(q)$.

Remark 5.7. We list the orders of the groups appearing in Lemma 5.6 according to [6]. We classify them into two cases depending on the arithmetic structure of the order.

Case 1.

$$
\begin{aligned}
& \left|A_{1}(q)\right|=\frac{1}{2} q\left(q^{2}-1\right) . \\
& \left|B_{n}(q)\right|=\frac{1}{2} q^{n^{2}} \Pi_{i=1}^{n}\left(q^{2 i}-1\right),(n \geq 2) . \\
& \left|C_{n}(q)\right|=\frac{1}{2} q^{n^{2}} \Pi_{i=1}^{n}\left(q^{2 i}-1\right),(n \geq 3) . \\
& \left|F_{4}(q)\right|=q^{24}\left(q^{12}-1\right)\left(q^{8}-1\right)\left(q^{6}-1\right)\left(q^{2}-1\right) . \\
& \left|G_{2}(q)\right|=q^{6}\left(q^{6}-1\right)\left(q^{2}-1\right) . \\
& \left|E_{7}(q)\right|=\frac{1}{2} q^{63}\left(q^{18}-1\right)\left(q^{14}-1\right)\left(q^{12}-1\right)\left(q^{10}-1\right)\left(q^{8}-1\right)\left(q^{6}-1\right)\left(q^{2}-1\right) . \\
& \left|E_{8}(q)\right|=q^{120}\left(q^{30}-1\right)\left(q^{24}-1\right)\left(q^{20}-1\right)\left(q^{18}-1\right)\left(q^{14}-1\right)\left(q^{12}-1\right)\left(q^{8}-1\right)\left(q^{2}-1\right) .
\end{aligned}
$$

Case 2.

$$
\begin{aligned}
& \left|{ }^{2} D_{n}(q)\right|=\frac{q^{n(n-1)}\left(q^{n}+1\right)}{\left(4, q^{n}+1\right)} \prod_{i=1}^{n}\left(q^{2 i}-1\right),(n \geq 4) . \\
& \left|D_{n}(q)\right|=\frac{q^{n(n-1)}\left(q^{n}-1\right)}{\left(4, q^{n}-1\right)} \prod_{i=1}^{n}\left(q^{2 i}-1\right),(n \geq 4) . \\
& \left|{ }^{3} D_{4}(q)\right|=q^{12}\left(q^{8}+q^{4}+1\right)\left(q^{6}-1\right)\left(q^{2}-1\right)=\frac{q^{12}}{q^{4}-1}\left(q^{12}-1\right)\left(q^{6}-1\right)\left(q^{2}-1\right) .
\end{aligned}
$$

Our next goal is to prove Lemma 5.9, which may be of independent interest. We need first an elementary observation, so we omit its proof.

Lemma 5.8. Let $q$ be an odd positive integer. If $r$ is an odd positive integer, then $\frac{q^{r}-1}{q-1}$ and $\frac{q^{r}+1}{q+1}$ are odd.

Lemma 5.9. Let $G$ and $A$ be finite groups of coprime orders and assume that $A$ acts on $G$ by automorphisms and let $C:=\mathbf{C}_{G}(A)$. If $G$ is a group appearing in Lemma 3.2, then $|G: C|$ is odd.

Proof. Let $|A|=r$. Since $(|A|,|G|)=1$, we have that $r$ is odd. By hypothesis $G$ is a simple group of Lie type defined over a field of $q$ elements, where $q$ is odd. The action can be assumed to be faithful; if not, the action of $A$ could be replaced by the action of $A / \mathbf{C}_{A}(G)$. Moreover, we can identify $A$ with the corresponding subgroup of $\operatorname{Aut}(G)$ and $A$ is $\operatorname{Aut}(G)$-conjugate to some group of field automorphisms of $G$. If we write $G=G(q)$, then $C=G\left(q_{0}\right)$, where $G\left(q_{0}\right)$ is the Lie group of the same type as $G$, but defined over the field of $q_{0}$ elements. Note that $q$ is odd and $q=q_{0}^{r}$. By Lemma 5.8

$$
\frac{q^{i}-1}{q_{0}^{i}-1}=\frac{\left(q_{0}^{r}\right)^{i}-1}{q_{0}^{i}-1}=\frac{\left(q_{0}^{i}\right)^{r}-1}{q_{0}^{i}-1}
$$

is odd. Assume that $G$ is a group appearing in Case 1 of Remark 5.7. Then the orders of $C$ and $G$ have the same arithmetic type and these orders are products of some $q^{i}-1$ and $q^{j}$, so it is not hard to check that $|G: C|$ is odd. Finally, since $q=q_{0}^{r}$ and $r$ are odd, one easily checks that $\left(4, q^{n} \pm 1\right)=\left(4, q_{0}^{n} \pm 1\right)$. From this we can get that $|G: C|$ is odd as well for all those groups appearing in Case 2 of Remark 5.7. 
The following result is also essential in the proof of Theorem C. It classifies those finite simple groups having a subgroup with prime power index. This is absolutely non-trivial and was established by Guralnick [7], and in fact, it was also used to prove Theorem 5.2.

Theorem 5.10. [7, Theorem 1] Let $G$ be a non-abelian simple group with $H<G$ and $|G: H|=p^{a}$, p prime. One of the following holds.

(a) $G=A_{n}$, and $H \cong A_{n-1}$, with $n=p^{a}$.

(b) $G=\operatorname{PSL}(n, q)$ and $H$ is the stabilizer of a line or hyperplane. Then $|G: H|=$ $\left(q^{n}-1\right) /(q-1)=p^{a}$. (Note that $n$ must be prime).

(c) $G=\operatorname{PSL}(2,11)$ and $H \cong A_{5}$.

(d) $G=M_{23}$ and $H \cong M_{22}$ or $G=M_{11}$ and $H \cong M_{10}$.

(e) $G=\operatorname{PSU}(4,2) \cong \operatorname{PSp}(4,3)$ and $H$ is the parabolic subgroup of index 27.

We are ready to prove Theorem $\mathrm{C}$, which we state again, but previously we remark that when $A=1$ (or the action is trivial), then the result is exactly Theorem 1.1 of [14].

Theorem 5.11. Suppose that a group $A$ acts coprimely on a group $G$. If $G$ has at most two $\mathbf{C}_{G}(A)$-orbits of non-nilpotent maximal $A$-invariant subgroups, then $G$ is solvable.

Proof. Let $G$ be a counterexample of minimal order. First we note that $G$ has no solvable non-trivial $A$-invariant normal subgroups; otherwise the fact that the hypothesis are inherited by $A$-invariant factors and the minimality of $G$ yield to the solvability of $G$. On the other hand, if all maximal $A$-invariant subgroups of $G$ are nilpotent, then either $G$ is nilpotent and we are finished or it is not, and then we apply Theorem 5.1 to get that $G$ is solvable. Henceforth, we can assume that $G$ has at least one non-nilpotent maximal $A$-invariant subgroup. Likewise, $G$ must have at least one nilpotent maximal $A$-invariant subgroup, say $M$; otherwise, all maximal $A$-invariant subgroups are non-nilpotent, so there are only two orbits of maximal $A$-invariant subgroups, and Theorem B implies that $G$ is solvable, a contradiction.

Next we prove that $M$ is a Sylow 2-subgroup of $G$. Notice that every non-trivial $A$ invariant Sylow subgroup of $M$ cannot be normal in $G$ and thus, its normalizer in $G$ is $M$ itself. We assert that $M$ is a Hall subgroup of $G$, for if $p$ were a common prime divisor of $|M|$ and $|G: M|$, then the Sylow $p$-subgroup of $M$ would be normal in $G$ since the normalizer in $G$ of a Sylow $p$-subgroup of $M$ would grow, a contradiction. Let $U$ be the $A$-invariant 2-complement of $M$, which is also a nilpotent Hall subgroup of $G$. Assume that $U \neq 1$. By a theorem of Wielandt (Satz IV.7.3 of [8]), there exists a normal subgroup $K$ of $G$ such that $G=M K$ and $M \cap K=1$. Clearly $K$ is $A$-invariant too. Let $q \in \pi(K)$ and $Q \in \operatorname{Syl}_{q}^{A}(K)$. By the Frattini argument $G=\mathbf{N}_{G}(Q) K$. Then $\mathbf{N}_{K}(Q)$ is normal in $\mathbf{N}_{G}(Q)$ and $\mathbf{N}_{G}(Q) / \mathbf{N}_{K}(Q) \cong G / K \cong M$. Thus $\mathbf{N}_{K}(Q)$ is an (A-invariant) normal Hall subgroup of $\mathbf{N}_{G}(Q)$, and by the Schur-Zassenhaus Theorem $\mathbf{N}_{G}(Q)$ has an $A$-invariant subgroup $M^{*}$, which is isomorphic to $M$. By another well-known theorem of Wielandt, as $M$ is a nilpotent Hall subgroup of $G$, all such Hall subgroups are conjugate in $G$, so we can apply Glauberman's Lemma to conclude that $M^{*}$ and $M$ are conjugate in $\mathbf{C}_{G}(A)$. In particular, $M^{*}$ is maximal $A$-invariant in $G$. Since $\left\langle M^{*}, Q\right\rangle=G$ and $Q$ is normalized by $M^{*}$ and $Q$, then $Q \unlhd G$, against the fact that $G$ has no solvable $A$-invariant normal subgroups. This contradiction proves that $U=1$, that is, $M \in \operatorname{Syl}_{2}(G)$, as required. 
We continue the proof by distinguishing two cases: whether $G$ has non-trivial proper $A$-invariant normal subgroups or not.

Case 1. We assume first that $G$ has no non-trivial proper $A$-invariant normal subgroup. Consequently $G=S_{1} \times \ldots \times S_{n}$ with $S_{i}$ non-abelian simple and isomorphic (possibly $n=1)$. Furthermore, we know that $A$ acts transitively on the set $\left\{S_{1}, \ldots, S_{n}\right\}$. Let $S:=S_{1}, B=\mathbf{N}_{A}(S)$ and $T$ a transversal of $B$ in $A$. We distinguish two subcases depending on whether $B$ acts trivially on $S$ or not.

a) Assume that $B$ acts trivially on $S$. First, we claim that $S$ has a maximal Sylow 2 -subgroup, say $P$. This will strongly reduce the possibilities for $S$. Indeed, if this is not the case, we can take a subgroup $H$ with $P<H<S$. Then we define $H_{0}=\prod_{t \in T} H^{t}$, which is $A$-invariant containing properly a Sylow 2 -subgroup of $G$. This contradicts the maximality of $M$, so the claim is proved. Therefore, according to the classification of the simple groups that posses maximal Sylow 2-subgroups (see [16]), it follows that $S \cong$ $\operatorname{PSL}(2, q)$ where $q$ is a Fermat or a Mersenne prime with $q \geq 17$.

We can assume that $n>1$ for if $n=1$, then the action of $A$ on $G$ is trivial and our result is exactly Theorem 1.1 of [14], so we are finished. Thus, by Lemma 4.1(a), we have $\mathbf{C}_{G}(A) \cong \mathbf{C}_{S}(B)=S<G$, and hence there exists a maximal $A$-invariant subgroup $K$ of $G$ with $\mathbf{C}_{G}(A)<K$ (observe that $K$ itself is a single $\mathbf{C}_{G}(A)$-orbit of non-nilpotent maximal $A$-invariant subgroups). We claim that $K$ does not contain any Sylow subgroup of $G$. Indeed, if $K$ contains an $A$-invariant Sylow $q$-subgroup, say $\hat{Q}$, of $G$ for a prime $q$, then we can write $\hat{Q}=\prod_{t \in T} Q^{t}$ for some $Q \in \operatorname{Syl}_{q}(S)$. But notice that $\left\langle Q^{S}\right\rangle=\mathbf{O}^{q^{\prime}}(S)=S$ because $S$ is simple, so we can apply Lemma 4.1(b) and get $G=\left\langle\mathbf{C}_{G}(A), \prod_{t \in T} Q^{t}\right\rangle \leq K$, a contradiction. This proves the claim. Now, let $L$ be a representative of the other $\mathbf{C}_{G}(A)$-orbit of non-nilpotent maximal $A$-invariant subgroups of $G$. From the hypotheses, the fact that the $A$-invariant Sylow 2 -subgroups of $G$ are the only nilpotent maximal $A$-invariant subgroups and the above property, we deduce that for every odd prime $p, L$ must contain an $A$-invariant Sylow $p$-subgroup of $G$, or equivalently, $|G: L|$ is a power of 2 . We consider the subgroup $S L$ (which is not $A$-invariant) and we have $|S: S \cap L|=|S L: L|$, which divides $|G: L|$. As a consequence $|S: S \cap L|$ is a power of 2. Moreover, $|S: S \cap L|>1$; otherwise, the fact that $A$ acts transitively on the $S_{i}$ implies that $L=G$, a contradiction. By applying Theorem 5.10 and according to the above paragraph, we conclude that $S \cong \operatorname{PSL}(2, q)$ with $q$ a Mersenne prime, and also $|S: S \cap L|=q+1=2^{a}$. Then $|S \cap L|=q(q-1) / 2$ is odd. Now, the maximal subgroups of $\operatorname{PSL}(2, q)$ are well-known (see for instance a Theorem of Dickson in [8]), and there exists a maximal (dihedral) subgroup $D$ in $S$ of order $q-1$ (an even number). Put $\hat{D}=\prod_{t \in T} D^{t}$ which is $A$-invariant and consequently, lies in some maximal $A$-invariant subgroup of $G$. Moreover, by order considerations, $\hat{D}$ contains an $A$-invariant Sylow $r$-subgroup of $G$ for every odd prime $r$ dividing $q-1$. This implies that $\hat{D}$ is not contained in $K$, so $\hat{D}$ must lie in some conjugate of $L$. Without loss, we may assume $\hat{D} \leq L$. But then $D \subseteq S \cap L$, contradicting the fact that $|S \cap L|$ is odd.

b) Assume that $B$ acts non-trivially on $S$. Since the alternating and the sporadic simple groups do not admit non-trivial coprime action, we have by using the CFSG that $S$ is a simple group of Lie type, defined over some finite field $F$. Also, the action of $B$ on 
$S$ can be assumed to be faithful; otherwise $B$ is replaced by $B / \mathbf{C}_{B}(S)$. Moreover, by replacing $B$ by a conjugate in $\operatorname{Aut}(S)$, we can assume that $B$ is induced by a group of automorphisms of the defining field $F$ of $G$. Write $|B|=r$. It follows that $|F|=q^{r}$ for some prime power $q$ and we write $S=S\left(q^{r}\right)$, that is, the simple group of Lie type " $S$ ". Then, it is known that $\mathbf{C}_{S}(B)=S(q)$, where $S(q)$ is the Lie group of the same type as $S$ defined over the field of $q$ elements.

Let $U \in \operatorname{Syl}_{2}^{B}(S)$. We claim that $U$ is a maximal $B$-invariant subgroup of $S$. Suppose not and take $W$ a maximal $B$-invariant subgroup of $S$ such that $U<W$. As above we construct $U_{0}=\prod_{t \in T} U^{t}$ and $W_{0}=\prod_{t \in T} W^{t}$. It is clear that $U_{0} \in \operatorname{Syl}_{2}^{A}(G)$, that $W_{0}$ is $A$-invariant and certainly $U_{0}<W_{0}$. This contradicts the maximality of $M$ and proves the claim. Furthermore, from the maximality of $U$ we deduce that $\mathbf{N}_{S}(U)=U$. Therefore, we can apply Lemma 5.8 to get that $\left|S: \mathbf{C}_{S}(B)\right|$ is odd. Accordingly, there should be some Sylow 2-subgroup of $S$ lying entirely in $\mathbf{C}_{S}(B)$. This contradicts the maximality of $U$ because $\mathbf{C}_{S}(B) \cong S(q)$ is not a Sylow 2-subgroup of $S$.

Case 2. Suppose that there exists a minimal $A$-invariant normal subgroup $N<G$. As the hypotheses are inherited by factor groups, by minimality, we easily have that $G / N$ is solvable. Moreover, $N$ is the only minimal $A$-invariant normal subgroup and $N$ cannot be solvable.

Now, let us take a maximal $A$-invariant subgroup $T$ of $G$ containing $N$, which is obviously non-nilpotent. By maximality of $M$, we have $G=M N$, so $|G: N|$ and in particular $|G: T|$ are powers of 2 . On the other hand, let $p \in \pi(N)$ an odd prime and let $P \in$ $\operatorname{Syl}_{p}^{A}(G)$. By the Frattini argument $\mathbf{N}_{G}(P) N=G$. As $P$ is not normal in $G$, there exists a maximal $A$-invariant subgroup, say $L_{p}$, containing $\mathbf{N}_{G}(P)$. Certainly $L_{p}$ does not contain $N$, so it lies in a distinct $\mathbf{C}_{G}(A)$-orbit of maximal subgroups that of $T$. Likewise, $L_{p}$ cannot contain any Sylow 2-subgroup of $G$ by the maximality of $M$. Therefore, the subgroups $L_{p}$ when $p$ runs in the set of odd primes of $\pi(N)$ constitute a single $\mathbf{C}_{G}(A)$-orbit of maximal $A$-invariant subgroups of $G$ different from those of $M$ and $T$. Also, all the subgroups $L_{p}$ must have index a power of 2 . Thus, we have proved that all non-nilpotent maximal $A$-invariant subgroups of $G$ have 2-power indexes, and Theorem 5.2 provides the final contradiction.

\section{REFERENCES}

[1] S. Adnan. On groups having exactly 2 conjugacy classes of maximal subgroups. II. Atti Accad. Naz. Lincei Rend. Cl. Sci. Fis. Mat. Natur. (8) 68, no. 3, 179, (1980).

[2] S. Adnan. On groups having exactly 2 conjugacy classes of maximal subgroups. Atti Accad. Naz. Lincei Rend. Cl. Sci. Fis. Mat. Natur. (8) 66, no. 3, 175-178, (1979).

[3] V.A. Belonogov. Finite groups with three classes of maximal subgroups. Math. USSR-Sb 59, no 1, 223-236, (1988).

[4] A. Beltrán and C.G. Shao. Restrictions on maximal invariant subgroups implying solvability of finite groups. Ann. Mat. Pura Appl (4) 198, 357-366, (2019).

[5] A. Beltrán and C.G. Shao. On the number of invariant Sylow subgroups under coprime action. $J$. Algebra 490 (15), 380-389, (2017).

[6] J.H. Conway, R.T. Curtis, S.P. Norton, R.A. Parker and R.A. Wilson. Atlas of finite groups. Oxford Univ. Press, London, (1985).

[7] R.M. Guralnick. Subgroups of prime power index in a simple group. J. Algebra, 1983, 81(2): 304-311.

[8] B. Huppert. Endliche Gruppen I. Springer, Berlin, (1967). 
[9] A.S. Kondriat'ev. Normalizers of the Sylow 2-subgroups in Finite Simple Groups. Math. Notes. 2005, 78(3-4), 338-346.

[10] A.S. Kondrat'ev. On prime graph components of finite simple groups. Mat. Sb. 180 (1989), 787-797.

[11] P. B. Kleidman and M. W. Liebeck. The subgroup structure of the finite classical groups, Cambridge University Press, Cambridge, (1990).

[12] H. Kurzweil and B. Stellmacher. The theory of finite groups. An introduction, Springer-Verlag, Berlin-Heidelberg-New York, (2004).

[13] M.W. Liebeck, C.E. Praeger, J. Saxl, The maximal factorizations of the finite simple groups and their automorphism groups. Mem. Amer. Math. Soc. 86, No. 432 (1990), 1-151.

[14] J.K. Lu and W. Meng. On finite groups with non-nilpotent subgroups. Monatsh. Math. 179, 99-103, (2014).

[15] J.K. Lu, L.N. Pang and X.G. Zhong. Finite groups with non-nilpotent maximal subgroups. Monatsh. Math. 171, 425-431, (2013).

[16] J.S. Rose. On finite insoluble groups with nilpotent maximal subgroups. J. Algebra 48, 182-186 (1977).

[17] D.L. Winter. The automorphism group of an extraspecial p-group. Rocky Mountain J. Math. 2, no 2, 159-168, (1972).

[18] A.V. Zavarnitsine. Recognition of the simple groups $L_{3}(q)$ by element orders, J. Group Theory, 7(1) :81-97(2004). 


\section{Appendix}

TABLE $2 . G=P S L_{n}(q)$

\begin{tabular}{|l|l|l|l|}
\hline Row & $M$ & $N$ & Remark \\
\hline 1 & $G L_{a}\left(q^{b}\right) \cdot b$ & $P_{1}, P_{n-1}$ & $a b=n, b$ prime \\
\hline 2 & $P S p_{n}(q) \cdot a$ & $P_{1}, P_{n-1}$ & $\begin{array}{l}n \geq 4 \text { even, } a=(2, q-1)(n / 2, q- \\
1) /(n, q-1) \text { is even }\end{array}$ \\
\hline 3 & $P S p_{n}(q) \cdot a$ & $S t a b\left(V_{1} \oplus V_{n-1}\right)$ & $\begin{array}{l}n \geq 4 \text { even, } a=(2, q-1)(n / 2, q- \\
1) /(n, q-1) \text { is even }\end{array}$ \\
\hline
\end{tabular}

TABle $3 . G=P S p_{2 m}(q), m \geq 2$

\begin{tabular}{|l|l|l|l|}
\hline Row & $M$ & $N$ & Remark \\
\hline 1 & $P S p_{2 a}\left(q^{b}\right) \cdot b$ & $P_{1}$ & $a b=m, b$ prime \\
\hline 2 & $S p_{2 a}\left(q^{b}\right) \cdot b$ & $O_{2 m}^{+}(q), O_{2 m}^{-}(q)$ & $q$ even, $a b=m, b$ prime \\
\hline 3 & $O_{2 m}^{-}(q)$ & $P_{m}$ & $q$ even \\
\hline 4 & $O_{2 m}^{-}(q)$ & $S p_{m}(q) \imath S_{2}$ & $m$ even, $q$ even \\
\hline 5 & $S z(q)$ & $O_{4}^{+}(q)$ & $\begin{array}{l}m=2, q=2^{f}, f \geq 3 \text { odd, two } \\
\text { classes of factorizations }\end{array}$ \\
\hline 6 & $G_{2}(q)$ & $O_{6}^{+}(q), O_{6}^{-}(q), P_{1}, N_{2}$ & $m=3, q$ even \\
\hline
\end{tabular}

TABle $4 . G=P S U_{n}(q), n \geq 3$

\begin{tabular}{|l|l|l|l|}
\hline Row & $M$ & $N$ & Remark \\
\hline 1 & $N_{1}$ & $P_{m}$ & $n=2 m$ \\
\hline 2 & $N_{1}$ & $P S p_{2 m}(q) . a$ & $n=2 m, a=(2, q-1)(m, q+1) /(n, q+1)$ \\
\hline 3 & 19.3 & $P_{1}$ & $n=3, q=8$ \\
\hline
\end{tabular}

TABLE 5. $G=\Omega_{2 m+1}(q), m \geq 3, q$ odd

\begin{tabular}{|l|l|l|l|}
\hline Row & $M$ & $N$ & Remark \\
\hline 1 & $N_{1}^{-}$ & $P_{m}$ & \\
\hline 2 & $G_{2}(q)$ & $P_{1}, N_{1}^{+}, N_{1}^{-}, N_{2}^{-}$ & $m=3$ \\
\hline 3 & $G_{2}(q)$ & $N_{2}^{+}$ & $m=3, q>3$ \\
\hline 4 & $P S p_{6}(q) \cdot a$ & $N_{1}^{-}$ & $m=6, q=3^{f}, a \leq 2$ \\
\hline 5 & $F_{4}(q)$ & $N_{1}^{-}$ & $m=12, q=3^{f}$ \\
\hline
\end{tabular}

TABLe $6 . G=P \Omega_{2 m}^{-}(q), m \geq 4$

\begin{tabular}{|l|l|l|l|}
\hline Row & $M$ & $N$ & Remark \\
\hline 1 & $P_{1}$ & $\hat{} G U_{m}(q)$ & $m$ odd \\
\hline 2 & $N_{1}$ & ${ }^{\wedge} G U_{m}(q)$ & $m$ even \\
\hline
\end{tabular}


TABLE $7 . G=P \Omega_{2 m}^{+}(q), m \geq 5$

\begin{tabular}{|l|l|l|l|}
\hline Row & $M$ & $N$ & Remark \\
\hline 1 & $N_{1}$ & $P_{m}, P_{m-1}$ & \\
\hline 2 & $N_{1}$ & ${ }^{\prime} G U_{m}(q) .2$ & $m$ even \\
\hline 3 & $N_{1}$ & $\begin{array}{l}\left(P S p_{2}(q)\right. \\
\left.P S p_{m}(q)\right) . a\end{array}$ & $m$ even, $q>2, a=g c d(2, m / 2, q-1)$ \\
\hline 4 & $N_{2}^{-}$ & $P_{m}, P_{m-1}$ & \\
\hline 5 & $P_{1}$ & ${ }^{\wedge} G U_{m}(q) \cdot 2$ & $m$ even \\
\hline 6 & $N_{1}$ & ${ }^{\wedge} G U_{m}(q) \cdot 2$ & if $m$ odd \\
\hline 7 & $\Omega_{9}(q) \cdot a$ & $N_{1}$ & $m=8, a \leq 2$ \\
\hline
\end{tabular}

